\title{
Dual inhibition of HDAC and tyrosine kinase signaling pathways with CUDC-907 attenuates TGF $\beta 1$ induced lung and tumor fibrosis
}

\author{
Wentian Zhang $\mathbb{D}^{1}$, Yajie Zhang ${ }^{1}$, Tian Tu${ }^{2}$, Sabastian Schmull ${ }^{3}$, Yu Han ${ }^{1}$, Wenbo Wang ${ }^{2}$ and Hecheng Li $^{1}$
}

\begin{abstract}
TGF $\beta 1$ signaling is a critical driver of collagen accumulation in pulmonary fibrotic diseases and a well-characterized regulator of cancer associated fibroblasts (CAF) activation in lung cancer. Myofibroblasts induced by TGF $\beta 1$ and other factors are key players in the pathogenesis of lung fibrosis and tumor. Tremendous attention has been gained to targeting myofibroblasts in order to inhibit the progression of fibrosis and myofibroblast-induced tumor progression and metastasis. Here we determined the therapeutic efficacy of simultaneously targeting PI3K and HDAC pathways in lung myofibroblasts and CAF with a single agent and to evaluate biomarkers of treatment response. CUDC-907 is a first-in-class compound, functioning as a dual inhibitor of HDACs and PI3K/AKT pathway. We investigated its effects in counteracting the activity of TGF $\beta 1$-induced myofibroblasts/CAF in regard to cell proliferation, migration, invasion, apoptosis in vitro antifibrosis efficiency in vivo. We found that CUDC-907 inhibited myofibroblasts/CAF cell proliferation, migration and apoptosis in a dose-dependent manner and caused cell cycle arrest at G1-S phase. CUDC907 not only inhibited myofibroblasts markers expression, but also significantly inhibited the phosphorylation level of AKT, mTOR, Smad2/3, and promoted acetylation of histones. Furthermore, the observed inhibitory effect was also confirmed in bleomycin-induced mice lung fibrosis and nude mouse transplanted tumor model. Overall, these data suggest that dual inhibition of HDAC and the tyrosine kinase signaling pathways with CUDC-907 is a promising treatment strategy for TGF $\beta 1$-induced lung and tumor fibrosis.
\end{abstract}

\section{Introduction}

Pathological lung fibrosis, and its promoting effects on tissue stiffness, is a major cause of human morbidity and mortality ${ }^{1}$. TGF- $\beta 1$ signaling is both an initiator and a driver of lung stiffness because of extracellular matrix (ECM) deposition and myofibroblasts differentiation. Approximately $80 \%$ of the upregulated genes in lungs of

\footnotetext{
Correspondence: Wenbo Wang (wangwenbo0903@126.com) or Hecheng Li (lihecheng2000@hotmail.com)

'Department of Thoracic Surgery, Ruijin Hospital, Shanghai Jiao Tong University School of Medicine, 197 Ruijin 2nd Rd, Shanghai 200025, China 2Department of Plastic and Reconstructive Surgery, Shanghai Ninth People's Hospital, Shanghai Jiao Tong University School of Medicine, Shanghai Key Laboratory of Tissue Engineering, 639 ZhiZaoJu Road, Shanghai 200011, China Full list of author information is available at the end of the article These authors contributed equally: Wentian Zhang, Yajie Zhang Edited by I. Amelio
}

patients with idiopathic pulmonary fibrosis are reported to be direct or indirect TGF- $\beta 1$ target genes ${ }^{2}$. Exaggerated TGF- $\beta 1$ signaling is strongly implicated not only in lung fibrotic diseases, but also in cancer progression ${ }^{3,4}$.

Myofibroblasts, induced by TGF $\beta 1$-mediated signaling, exhibit continued activation in proliferation, anti-apoptosis, migration, invasion as well as expressing collagens and myofibroblasts markers such as $\alpha$-SMA, FAP- $\alpha$, and PDGFR etc ${ }^{4-6}$. Myofibroblasts in tumor are generally referred as tumor-associated fibroblasts or cancerassociated fibroblasts (CAF) and contribute as key fibro genic cells aggravating non-small cell lung cancer (NSCLC) growth and progression ${ }^{7,8}$. Thus, intervening myofibroblast-induced pro-fibrotic and pro-tumorigenic activities using signaling inhibitor can be an interesting approach against fibrosis and cancer.

\section{(-) The Author(s) 2020}

(c) (i) Open Access This article is licensed under a Creative Commons Attribution 4.0 International License, which permits use, sharing, adaptation, distribution and reproduction cc) in any medium or format, as long as you give appropriate credit to the original author(s) and the source, provide a link to the Creative Commons license, and indicate if changes were made. The images or other third party material in this article are included in the article's Creative Commons license, unless indicated otherwise in a credit line to the material. If material is not included in the article's Creative Commons license and your intended use is not permitted by statutory regulation or exceeds the permitted use, you will need to obtain permission directly from the copyright holder. To view a copy of this license, visit http://creativecommons.org/licenses/by/4.0/. 
Among multiple intracellular signaling pathways, activation of PI3K/Akt/mTOR pathway has been demonstrated to enhance inflammation, pro-survival activation in tumor cells, and deposition of ECM component ${ }^{9}$. A recent study demonstrated comparatively higher level of total and phosphorylated forms of Rapamycin (mTOR) in myofibroblasts ${ }^{10}$. Investigation on several mTOR inhibitors suggested that PI3K/Akt/mTOR pathway might be a potential target for anti-fibrosis therapy ${ }^{11}$. Besides, aberrant expression of histone deacetylases (HDACs) is frequent in human cancers and may participate in the progression of fibrotic development in idiopathic pulmonary fibrosis (IPS) and tumor progression ${ }^{12-14}$. Since HDAC inhibitors were widely exploited for their antifibrosis effects on liver/kidney fibrosis and their anticancer roles $^{15,16}$, they may become other promising therapeutic drugs for lung and tumor fibrosis ${ }^{17}$.

Hence, CUDC-907, a newly-synthesized small molecular compound that has been designed by incorporating HDAC inhibitory functionality into a PI3K inhibitor pharmacophore comes into our sight ${ }^{18,19}$, while its impacts on fibrosis are not clear. It was reported that CUDC-907 exerts a potent anti-tumor activity against B cell lymphoma and other tumor cell lines ${ }^{18}$. Recently, a phase-I clinical trial on its biosafety, tolerability, and preliminary activity revealed its bright future in clinical application ${ }^{20}$.

This study investigated the anti-fibrotic effect of CUDC907 using in vitro cell culture, in vivo xenograft model, which demonstrated that CUDC-907 could inhibit the proliferation, migration, invasion, and ECM deposition of in vitro cultured myofibroblasts and could also suppress collagen accumulation and tumor growth in vivo.

\section{Materials and methods}

\section{Cell lines, cell culture, reagents, and CAF isolation}

The fibroblast cell line NIH3T3 and human lung fibroblasts were purchased from Cell Bank of the Chinese Academy of Sciences (Shanghai, China) where they were characterized by mycoplasma detection, DNA -fingerprinting, isozyme detection, and cell vitality detection. These cell lines were immediately expanded and frozen such that they could be restarted every 6 months from a frozen vial of the same batch of cells. CUDC-907, GDC-0941 (PI3K/Akt/mTOR inhibitor), and Trichostatin A (HDAC inhibitor) solid powder was purchased from Selleck Chemicals (Houston, TX, USA). The compound powder was dissolved in pure dimethyl sulfoxide (DMSO) to make a concentration of $10 \mathrm{mM}$ for storing under condition at $4{ }^{\circ} \mathrm{C}$. Before using, the CUDC-907 stock solution was diluted in Dulbecco's modified eagle medium (DMEM, Hyclone, Logan City, UT, USA) to result in designed drug concentrations with DMSO volume identical to that of vehicle control group.
Table 1 Characteristic of patients.

\begin{tabular}{lllll}
\hline & Sex & Age & TNM stage & Pathological type \\
\hline CAF1 & Female & 72 & T1bN1M0 & Adenocarcinoma \\
CAF2 & Male & 64 & T2N1M0 & Squamous cell carcinoma \\
\hline
\end{tabular}

The final concentration of DMSO solvent was controlled under $0.001 \%(v / v)$ during each of following assays.

The cell lines were maintained in DMEM with $4.5 \mathrm{~g} / \mathrm{L}$ of glucose, $2 \mathrm{mmol} / \mathrm{L}$ of $\mathrm{L}$-glutamine, and $110 \mathrm{mg} / \mathrm{L}$ of sodium pyruvate, supplemented with $10 \%$ fetal bovine serum (FBS), and Antibiotic-Antimycotic (100×, Gibco, Grand Island, NY, USA) in a standard humidified incubator at $37{ }^{\circ} \mathrm{C}$ in $5 \% \mathrm{CO}_{2}$ and $95 \% \mathrm{O}_{2}$ atmosphere.

Human lung tissue samples were donated by 2 NSCLC patients (Table 1, Supplementary Fig. 1) and were collected according to a clinical protocol at Ruijin Hospital. This protocol was approved by the Ethics Committee of Ruijin Hospital (KY 2018-104). All patients were enrolled after written informed consent. Tissue samples were stored at $-80^{\circ} \mathrm{C}$ until their use for experiments.

Isolation and culture of fibroblasts were performed according to a previous protocol ${ }^{21}$. In brief, lung tissue samples were collected in sterilized $10-\mathrm{ml}$ centrifuge tubes after surgical excision and then immersed and washed in HANK's solution for 3 times with 5 min each time. Sterilized scalpels and scissors were used to mince the tissue into small pieces. These small pieces were treated with sufficient $0.25 \%$ collagenase (dissolved in Hank's balanced salt solution, Gibco, Grand Island, NY, USA) for $1 \mathrm{~h}$ at $37^{\circ} \mathrm{C}$ on a shaker. After digestion, cells were collected via centrifugation at 1500 RPM for $5 \mathrm{~min}$ and resuspended in DMEM supplemented with $10 \%$ fetal bovine serum (FBS, Hyclone) and Antibiotic-Antimycotic. Cells were seeded on a $10-\mathrm{cm}$ culture dish at a density of $2 \times 10^{4} / \mathrm{cm}^{2}$ and cultured in a $37^{\circ} \mathrm{C}$ thermostat incubator containing $5 \% \mathrm{CO}_{2}$ atmosphere. Cells were passaged at the same density before they reached $90 \%$ of confluence. The second passage cells were used in this research.

\section{CCK-8 cell proliferation analysis}

Cell proliferation experiments were performed using the cell counting kit-8 (CCK-8; Dojindo, Japan). Cells were seeded at a density of 3000 cells $/ 100 \mu \mathrm{L}$ in five copies in 96-well opaque plates with clear bottoms (Falcon). The next day, cells were supplemented with $100 \mu \mathrm{L}$ of fresh medium containing the indicated concentrations of CUDC-907 with or without $5 \mathrm{ng} / \mathrm{mL}$ TGF $\beta 1$. Same procedure was used when treating cells with $30 \mathrm{nM}$ CUDC907 or GDC-0941 or Trichostatin A, respectively. Medium with drug/vehicle was replenished every $48 \mathrm{~h}$. CCK-8 solution was diluted in DMEM to obtain volume ratio as 
1:10 before the test, and then the medium was replaced by $100 \mu \mathrm{L}$ mixed liquid in each well tested. After incubation in a $37{ }^{\circ} \mathrm{C}$ thermostat incubator containing $5 \% \mathrm{CO}_{2}$ atmosphere for $4 \mathrm{~h}$, the medium was collected for measuring the optical density values at $450 \mathrm{~nm}$ using a microplate reader (Thermo Scientific, San Jose, CA, USA). Each assay was conducted and repeated for three independent pooled cell samples.

\section{Cell viability analysis}

Live/Dead Viability/Cytotoxicity Kit (Invitrogen, Carlsbad, CA, USA) was used to evaluate cell viability. At day 3, the 96-well plates containing NIH3T3 cells treated with drug as shown above were incubated with a solution of $2 \mathrm{mM}$ calcein-acetoxymethyl (Calcein-AM, R\&D, Minneapolis, Canada) and $1 \mu \mathrm{g} / \mathrm{ml}$ propidium iodide (PI, Sigma-Aldrich, USA) for $30 \mathrm{~min}$. Then reagents were washed out by PBS and plates were taken for phase contrast microscopy for assessment of gross features. In the Live/Dead assay, calcein-AM (GREEN) is a fluorescent dye that is activated by intracellular esterases, which is trapped inside the intact membrane of live cells. PI is a red fluorescent dye, which is impermeable to an intact cell membrane.

\section{Cell cycle and apoptosis analysis}

Cells were plated at a density of $2 \times 10^{5}$ in a six-well plate and treated with vehicle or CUDC-907 (10 nM and $30 \mathrm{nM})$ with or without TGF $\beta 1$ for $48 \mathrm{~h}$. Subsequently, cells were detached with $0.25 \%$ trypsin-EDTA (Gibco), centrifuged and resuspended in phosphate buffered saline (PBS) in a 1.5-ml Eppendorf tube followed by 3 washes in PBS.

To examine the cell cycle profiles, cells were subsequently centrifuged at $1500 \mathrm{RPM}$ for $5 \mathrm{~min}$ and then resuspended and fixed in $1 \mathrm{~mL}$ ice-cold $70 \%$ ethanol for $24 \mathrm{~h}$. Afterward, fixed cell samples were centrifuged and washed in PBS. Cell pellets were stained using a cell cycle analysis kit (7sea Phamatech, Shanghai, P.R. China) and incubated at $37^{\circ} \mathrm{C}$ for $30 \mathrm{~min}$. Flow cytometer (Beckman Coulter, CA, USA) combined with ModiFit LT v2.0 software was applied in flow cytometric analyses.

To examine apoptosis, cells were resuspended and stained using Alexa Fluor 488 Annexin V/PI Apoptosis Kit (ThermoFisher Scientific, USA) and incubated at room temperature for $15 \mathrm{~min}$. Then cells were analyzed by flow cytometry and fluorescence emission was measured at $530 \mathrm{~nm}$. Each assay was repeated in three independent cell samples.

\section{In vitro cell migration assay}

Scratch assay was employed to analyze the suppressive effect of CUDC-907 on migratory ability of NIH3T3 cells and CAF1 and CAF2. After over 90\% confluence in sixwell plates, the cell monolayer was scratched using a 200- $\mu \mathrm{l}$ pipette tip and then cultured in serum-free medium with CUDC-907 $(10 \mathrm{nM}$, and $30 \mathrm{nM}$ with or without TGF $\beta 1$ ) for $24 \mathrm{~h}$. Photographs were taken at $0 \mathrm{~h}, 24 \mathrm{~h}$ after scratching and blank areas were measured by Image-Pro Plus (Version 6.0, Media Cybernetics, Maryland, USA). Percentages of area filled by cells were calculated according to the formula below: Cell migration rate $(\%)=$ (Area0h - Area24h) $/$ Area0h $\times 100 \%$. Three replicates were set in each group and normalized to DMSO group.

Subsequently, a transwell system (Corning, 8- $\mu \mathrm{m}$ pore size) was utilized for migration assay. Briefly, $3 \times 10^{4}$ starved CAF1 suspended in $150 \mu \mathrm{l}$ serum-free medium with CUDC-907 $(10 \mathrm{nM}$ and $30 \mathrm{nM}$ with or without TGF $\beta 1$, respectively) were seeded in the upper compartment of the Boyden chamber in a 24-well plate. Lower compartment was filled with $500 \mu \mathrm{l}$ DMEM with $10 \%$ FBS or pre-seeded with lung cancer cell lines, respectively. After 24-h incubation, a cotton swab was used to wipe non-migrated cells up on the top surface of the membrane, and the migrated cells on bottom surface was fixed in $4 \%$ paraformaldehyde and stained with 4',6-diamidino2-phenylindole (DAPI, Sigma, USA). The migrated cells in five randomly selected high-power fields were counted using Image-Pro Plus. This assay was repeated for three pooled cell samples.

\section{RNA extraction and complementary DNA synthesis}

Total RNA was extracted from cells using Trizol reagent (Invitrogen Life Technologies Inc., NY, USA). RNA purity and concentration were confirmed via DU800 spectrophotometer (Beckman Coulter). cDNA was synthesized using AMV reverse transcriptase (Promega, WI, USA) with $2 \mu \mathrm{g}$ of total RNA according to the manufacturer's instruction as previously described ${ }^{22} 22$

\section{Real-time quantitative polymerase chain reaction}

Gene expression level was evaluated by quantitative real-time PCR (qPCR) analysis with Power SYBR Green PCR master mix $(2 \times)$ (Applied Biosystems, Foster City, CA, USA). qPCR was conducted in a real-time thermal cycler (Stratagene Mx3000PTM QPCR System, La Jolla, CA, USA) according to the protocol published ${ }^{22}$. qPCR was performed as follows: $95^{\circ} \mathrm{C}$ for $10 \mathrm{~min}$ then 40 cycles $\left(95^{\circ} \mathrm{C}\right.$ for $30 \mathrm{~s}, 58^{\circ} \mathrm{C}$ annealing temperature for $30 \mathrm{~s}$ and $72^{\circ} \mathrm{C}$ for $45 \mathrm{~s}$ ). Human housekeeping gene GAPDH was employed as an internal control. Each assay was performed in triplicate and all experiments were repeated in at least three different cell samples. The human primers for real-time qPCR analysis and their annealing temperatures are displayed in Table 2.

\section{Western blotting and p-Akt ELISA assay}

Total protein was extracted from CAF1 treated as mentioned above by using RIPA lysis buffer plus $1 \%$ 
Table 2 Primers used in quantitative PCR analysis.

\begin{tabular}{|c|c|c|c|}
\hline Gene & Primer Sequence $\left(5^{\prime}-3^{\prime}\right)$ & Annealing temperature $\left({ }^{\circ} \mathrm{C}\right)$ & Product size (bp) \\
\hline Collagen I & $\begin{array}{l}\text { Sense: GGCGGCCAGGGCTCCGACCC } \\
\text { Antisense: AATTCCTGGTCTGGGGCACC }\end{array}$ & 58 & 347 \\
\hline a-SMA & $\begin{array}{l}\text { Sense: CATCATGCGTCTGGATCTGG } \\
\text { Antisense: GGACAATCTCACGCTCAGCA }\end{array}$ & 58 & 107 \\
\hline TGFß1 & Sense: AAGGACCTCGGCTGGAAGTG Antisense: CCGGGTTATGCTGGTTGTA & 58 & 136 \\
\hline Collagen III & $\begin{array}{l}\text { Sense: TGGTGTTGGAGCCGCTGCCA } \\
\text { Antisense: CTCAGCACTAGAATCTGTCC }\end{array}$ & 58 & 373 \\
\hline
\end{tabular}

PMSF and 1\% protein phosphatase inhibitor. Cell lysates were resolved on SDS-PAGE gels and then transferred to PVDF membranes. Immunoblotting was performed using standard procedures published ${ }^{23}$. The membranes were probed with the following primary antibodies against: Fibronectin (ab32419, Abcam, USA), Collagen III (ab184993, Abcam, USA), FAP(\#66562, Cell signaling Technology, USA), BMP4(ab39973, Abcam, USA), PDGFR(\#3174, Cell signaling Technology, USA), PDK1 (\#5662, Cell signaling Technology, USA), AKT(\#4691, Cell signaling Technology, USA), p-AKT(Ser473)(\#4060, Cell signaling Technology, USA), mTOR(\#2983, Cell signaling Technology, USA), p-p70S6(T389)(\#9234, Cell signaling Technology, USA), Smad2/3(\#8685, Cell signaling Technology, USA), HDAC1-6(\#5356, \#5113, \#3949, \#3443, \#7558, Cell signaling Technology, USA), Acetyl-Histone H3(\#4243, Cell signaling Technology, USA), $\beta$-actin(\#4970, Cell signaling Technology, USA). Then, the membranes were subsequently incubated with secondary antibody (Anti-rabbit IgG \#7074, Anti-mouse IgG, \#7076, Cell signaling Technology, USA) coupled with Horseradish Peroxidase (HRP). Enhanced Chemiluminescence (ECL) detection was applied to visualize the protein band.

To further understand the effect of CUDC-907 in regulating Akt phosphorylation, CAF1 were seeded at a density of $2 \times 10^{6}$ in a $9 \mathrm{~cm}^{2}$ dish plate and treated with vehicle or CUDC-907 (10 nM and $30 \mathrm{nM})$ with or without TGF $\beta 1$ and collected at different time points $(4 \mathrm{~h}, 8 \mathrm{~h}, 12 \mathrm{~h}$, $24 \mathrm{~h}, 48 \mathrm{~h}$ ). p-Akt levels of cell lysates were analyzed by using a Akt(Phospho) [pT308] Multispecies InstantOne ELISA kit(\#85-86044-11, Thermofisher science, USA).

\section{Animal studies}

C57BL/6 male mice and nude male mice aging from 6 to 8 weeks were purchased from Shanghai Slaccas experimental animal limited liability company. All animal experimental protocols were approved by the Animal Care \& Welfare Committee of Shanghai ninth people's hospital affiliated to Shanghai Jiaotong University School of medicine. C57BL/6 mice were assigned to one of the following three treatment group: (1) controls receiving intratracheal instillation of $\mathrm{NaCl} 0.9 \%$ solution; (2) mice treated with intratracheal instillation of bleomycin $(30 \mu \mathrm{L}$ $2 \mathrm{mg} / \mathrm{kg}$ ) on day 0 and then treated as a control with $30 \%$ Captisol by oral gavage; (3) mice treated with bleomycin on day 0 and then treated as a treatment group from day 3, which was administered $50 \mathrm{mg} / \mathrm{kg}$ CUDC-907(resolved in 30\% Captisol) by oral gavage, on a 5-day on/2-day off dosing regimen. Body weight was measured weekly and mice were sacrificed at day 18 . The lungs were lavaged, and then snap-frozen in liquid nitrogen for hydroxyproline assay, or $4 \%$ paraformaldehyde fixed, paraffin embedded, for immunohistochemistry.

To verify the effect of CUDC-907 in regulating CAF in lung cancer, xenograft model was used. A549 cell line and CAF1 expressing luciferase (3:2) mixtures were injected subcutaneously into axilla of mice to establish the NSCLC model. After tumor formation (Day 10), mice were divided randomly into two groups: control, CUDC-907 treatment. The mice in control group were treated with $30 \%$ Captisol and mice in treatment group were treated with $50 \mathrm{mg} / \mathrm{kg}$ CUDC-907 (resolved in 30\% Captisol) by oral gavage, on a 5-day on/ 2-day off dosing regimen. Fluorescein is a compound for bioluminescence imaging (BLI). At Day 10, 25, and 31, mice were anesthetized with isoflurane and then injected with $75 \mathrm{mg} / \mathrm{kg}$ D-luciferin solution (MCE, Monmouth Junction, USA) for imaging. The photos of mice were obtained from Image Studio Instrument (Lincoln, Nebraska, USA). Body weight and tumors were measured every three days. After euthanasia, primary tumor tissues were snap-frozen and some primary tumors were $4 \%$ paraformaldehyde fixed, paraffin embedded, and sectioned for immunohistochemistry. No blinding was done for animal studies.

\section{Histologic analysis}

Left lung and tumor sections were stained with hematoxylin and eosin (HE) and Masson's trichrome staining was performed to assess the formation of lung collagen. 
The whole section was imaged with a NIKON ECLIPSE $\mathrm{C} 1$ microscope and tiled using 10\% image overlap into a single panoramic by NIKON DS-U3 software (NIKON).

\section{Hydroxyproline assay}

Lung collagen content was evaluated using hydroxyproline assay. Briefly, tissue was minced and hydrolyzed in $1 \mathrm{ml} 12 \mathrm{~N} \mathrm{HCl}$ at $100^{\circ} \mathrm{C}$ for $20 \mathrm{~min}$, the hydroxyproline was detected by incubation with chloramine $\mathrm{T}$ and $\mathrm{p}$ dimethylaminobenzaldehyde, and the absorbance was measured at $550 \mathrm{~nm}$. Each sample was run in triplicate. Collagen content in lung was expressed as micrograms of collagen per lung and was converted from micrograms of hydroxyproline.

\section{Immunohistochemical and immunofluorescence staining}

Lung and tumor specimens were fixed in $4 \%$ paraformaldehyde at $4{ }^{\circ} \mathrm{C}$ overnight and then embedded in paraffin. Sections were deparaffinized, serially rehydrated, treated with $1 \times$ citrate buffer at $120^{\circ} \mathrm{C}$ for antigen retrieval, blocked, and then immunostained with antibodies (Information showed in Western blotting) over night at $4{ }^{\circ} \mathrm{C}$. 3,3'-diaminobenzidine (DAB, Servicebio, G1211) with GTVision ${ }^{\mathrm{TM}}$ III detection system/Mo\&Rb (Dako, K5007) was used to detect immunoreactivity of the primary antibody. Slides were scanned at $20 \times$ magnification using CICXSP-C204 microscope (NIKON).

Paraffin embedded sections were also stained with $\alpha$ SMA antibody and IgG isotype controls. Where indicated in the figure legends, mosaic images were generated from multiple $\times 20$ images captured on a NIKON ECLIPSE C1upright fluorescent microscope and tiled using 10\% image overlap by NIKON DS-U3 software (NIKON).

\section{Statistical analysis}

All experiments were repeated at least three times. All the data were presented as mean \pm standard deviation (SD). Unpaired $t$ test One-Way ANOVA was used to judge if there was a significant difference among multiple groups, and subsequently $p$ values were calculated by LSD test. All statistical calculations were conducted via the software SPSS (version 22.0, SPSS Inc., IL, USA). Difference was considered significant when $p$ values were $<0.05$.

\section{Results}

\section{CUDC-907 inhibits cellular proliferation and causes G1/S arrest and apoptosis}

We first investigated the effect of CUDC-907 treatment on cellular proliferation using 4 different fibroblast origin: 2 fibroblast cell lines and 2 primary lung CAF from squamous and adenocarcinomas [Table 1, Supplementary Fig. 1]. We found that CUDC-907 treatment significantly inhibited cellular proliferation in a time- and dose- dependent manner and caused cell death at higher concentrations regardless of TGF $\beta 1$ stimulation (Fig. 1a-f). These results were validated by Calcein AM/PI fluorescent staining (Fig. 1i-k). Furthermore, we compared the anti-proliferate effect of CUDC-907 with PI3K inhibitor GDC0941 and HDAC inhibitor Trichostatin A for CUDC-907 was designed based on their active ingredients (Fig. 11) ${ }^{19}$. We found that CUDC-907 showed higher efficiency in inhibiting fibroblasts proliferation than GDC0941 and Trichostatin A at the same dose.

To understand the mechanism by which CUDC-907 treatment inhibited cellular proliferation, we tested it effect on cell cycle progression and apoptosis. We treated NIH-3T3 and HLF1 cell lines with CUDC-907 for $24 \mathrm{~h}$ and observed G1-S arrest post drug treatment (Fig. 2a, b and Supplementary Fig. 2).

We next evaluated the effect of CUDC-907 treatment on apoptosis using the Annexin V/PI assay and found increased activity at $24 \mathrm{~h}$ that was dose dependent (Fig. 2d, f). To further determine the effect of CUDC-907 treatment in regulating myofibroblasts, we stimulated HLF1 with TGF $\beta 1$ and subsequently added CUDC-907. We found that CUDC-907 treatment significantly increased apoptosis in a dose-dependent manner (Fig. 2e, g).

\section{CUDC-907 inhibits cell migration and invasion}

One of the hallmarks of fibroblasts is their ability to respond to TGF $\beta 1$ and become activated, resulting in enhanced properties of proliferation, migration and production of growth factors and extracellular matrix (ECM) which is the biology and function of fibroblasts in cancer In order to investigate the suppressive effect of CUDC907 on myofibroblasts migration capacity, in vitro scratched assay and migration assay were employed. As shown, at $24 \mathrm{~h}$ post scratching, CUDC-907 treatment significantly decreased cellular migration and invasion in all four fibroblast sources with or without TGF $\beta 1$ stimulation (Fig. 3a, b). We next evaluated the effect of CUDC-907 treatment on CAF1 cocultured with lung cancer cell lines. In the transwell migration assay, four lung cancer cell lines were seeded in down chamber and migrated cells decreased in a dose-dependent manner with CUDC-907 treatment (Fig. 4a, b).

We further evaluated the effect of CUDC-907 treatment on proteins known to regulate fibroblasts migration and invasion. Vimentin is recognized as a major type III intermediate filament protein participates in cell adhesion, migration and invasion ${ }^{24}$. Another protein is Vinculin that is present in cell-cell junctions and plays a key role not only in the generation of traction forces, but also in directional migration of cells ${ }^{25,26}$. We found that the protein expression of Vimentin was reduced with CUDC907 treatment, but there was a modest compensatory increase in vinculin protein levels in CAF1 (Fig. 4e). 


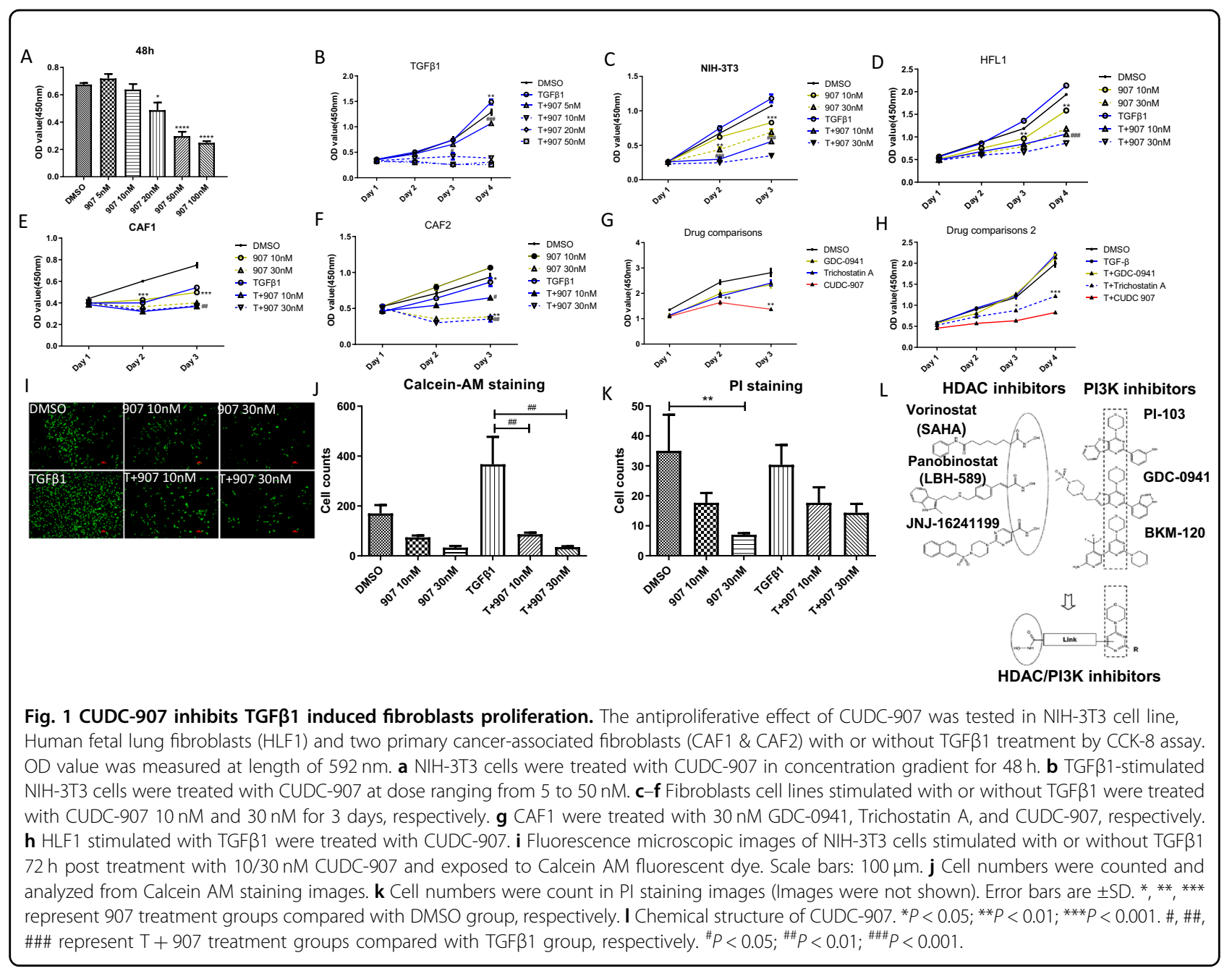

\section{CUDC-907 attenuated TGF $\beta 1$-induced fibroblast differentiation and collagen expression}

In lung cancer fibrosis, persistent emergence and accumulation of cancer cells in a given tissue represents an ongoing tissue injury, initiating a chronic wound healing response towards the cancer cells. CAF are largely governed by the growth factors released by cancer cells. Among these factors, TGF $\beta$ is one of the most important key mediators of CAF activation.

To test antifibrotic activity of CUDC-907, we evaluated whether CUDC-907 treatment influenced CAF differentiation and collagen expression under TGF $\beta$ stimulation. CUDC-907 treatment significantly inhibited collagen, fibronectin, PDGFR, and PDK1 (Fig. 5a, d). We next evaluated the effect of CUDC-907 treatment on proteins known to regulate CAF differentiation. We found that protein expression of FAP, PDGFR, and PDK1 was decreased, especially at the concentration of $30 \mathrm{nM}$ (Fig. 5a). To understand the mechanism of CUDC-907 in regulating TGF $\beta$-induced tumor fibrosis, we evaluated PI3K/AKT signaling protein levels of TGF $\beta 1$-stimulated
CAF1 treated with CUDC-907. As expected, we found that CUDC-907 treatment effectively reduced phosphoAKT, phosphor-p70S6, phosphor-mTOR, and Smad2/ 3levels (Fig. 5b, c).

We also evaluated the effect of CUDC-907 as a histone deacetylase inhibitor in CAF1 and the specific HDAC it targets. CUDC-907 treatment in CAF1 resulted in increased acetylation of histone 3, consistent with its effect as a histone deacetylase inhibitor (Fig. 5c). However, the effect of CUDC-907 on specific HDAC protein levels is unknown. Because HDAC1, HDAC2, HDAC3, HDAC5and HDAC6 have been reported to be overexpressed in CAF, we evaluated the effect of CUDC-907 treatment on these specific HDAC proteins. We found that CUDC-907 treatment reduced TGF $\beta 1$-induced HDAC1, HDAC2, HDAC3, HDAC5, and HDAC6 (Fig. 5c).

CUDC-907 attenuated collagen expression and production of in bleomycin-induced lung fibrosis in mice

Whether CUDC-907 could attenuate ECM deposition is essential for assessing its anti-fibrosis effect. Thus, we 


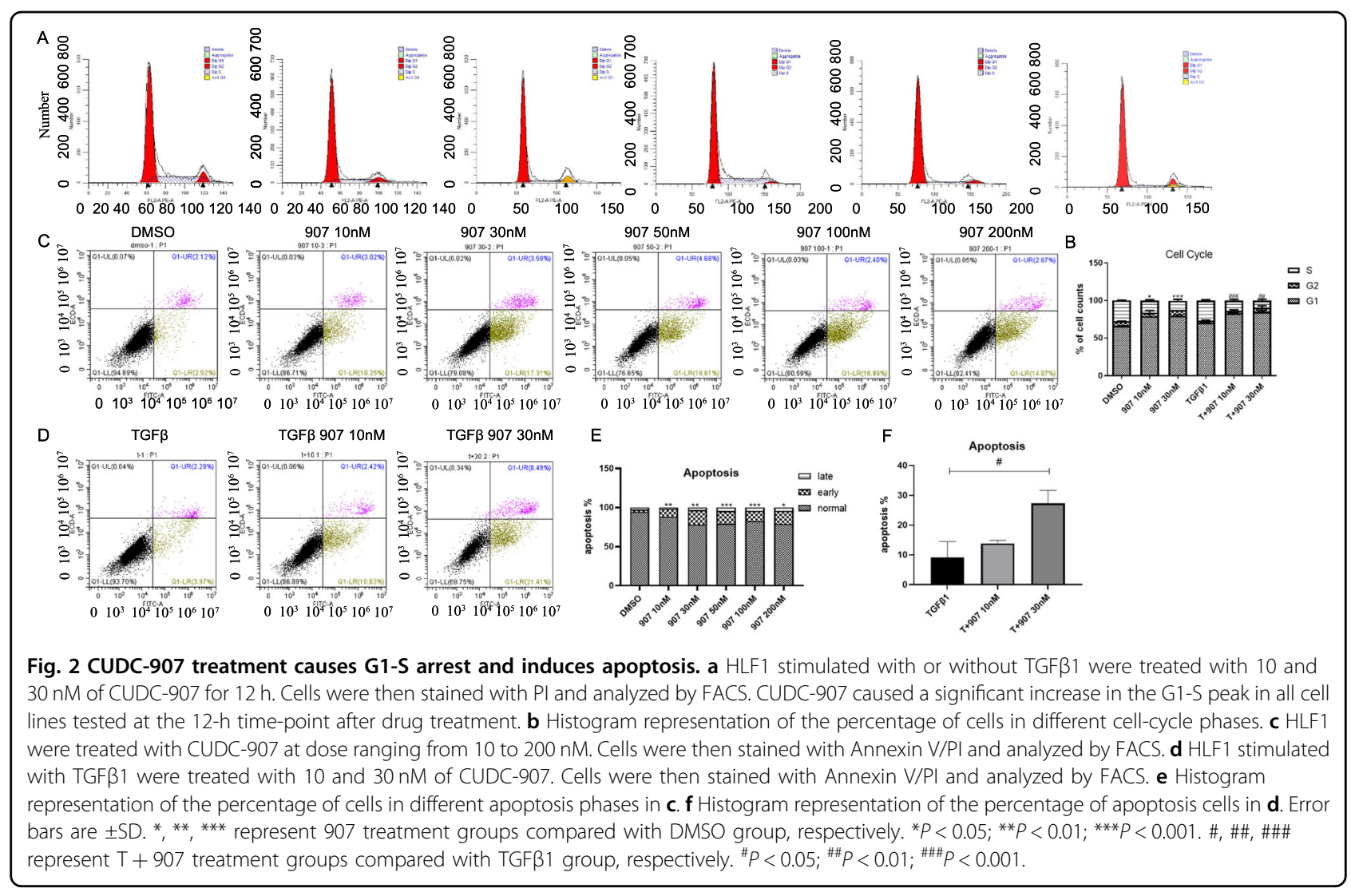

evaluated the in vivo effect of clinical-grade CUDC-907 in a fibrosis mouse model that recapitulates the clinical behavior of idiopathic pulmonary fibrosis. Mice were treated by either 35\% Captisol (control) or $1 \mathrm{mg} / \mathrm{kg}$ CUDC-907 dissolved in 35\% Captisol after intratracheal bleomycin injection (Fig. 6g). We found that CUDC-907 treatment inhibited collagen accumulation (Fig. 6a, c, d). At day 18 these mice exhibited marked attenuation of bleomycin-induced total lung collagen deposition (Fig. 6b). Furthermore, immunohistochemical staining of left lung sections showed that CUDC-907 treatment inhibits bleomycin-induced total Col1, Col3, and a-SMA (Fig. 6f).

\section{CUDC-907 inhibits tumor growth and fibrosis in a human xenograft mouse model constituting both CAF1 and A549 cell line}

The effectiveness of CUDC-907 was also tested in a classic xenograft model as illustrated in Fig. $7 \mathrm{~b}$ by injecting A549 cells subcutaneously. In order to assess CUDC-907 treatment in inhibiting tumor fibrosis, we mixed CAF1 with A549 human adenocarcinoma cells in a $3: 2$ ratio and inoculated these mixtures subcutaneously in immune-deficient nude mice. Luciferase was induced into CAF1s to allow their detection in vivo.
At day 25 and day 31 after drug administration, CUDC907 treatment resulted in significantly less overall tumor burden in both CAF1 presence and absent mouse models (Fig. 7a, d). Furthermore, numbers of CAF1 was significantly decreased in the CUDC-907 treatment group (Fig. 7c). Masson's trichrome staining, IF and IHC showed significantly reduced collagen accumulation, as well as aSMA and Collagen 3 expression (Fig. 7e, f, g).

\section{Discussion}

In this study, we evaluated the antifibrosis activity of CUDC-907, a first-in-class dual PI3K, and HDAC inhibitor, in in vitro and in vivo studies. We found that CUDC-907 inhibits TGF $\beta 1$-induced fibroblasts proliferation, migration. Moreover, it effectively inhibits commonly activated signaling pathways in lung and tumor fibrosis, and induces G1-S arrest and apoptosis. We also found that HDAC2 is highly expressed after TGF $\beta 1$ stimulation and this effect could be prevented via CUDC-907 treatment. Thus, our data show that CUDC-907 is a promising candidate against fibrosis and tumor progression.

TGF $\beta 1$ has been shown to be a key modulator in the synthesis of ECM, and also stimulates various intracellular pathways that can promote cell viability. Although attractive as a target, the advantage of TGF $\beta 1$ in 


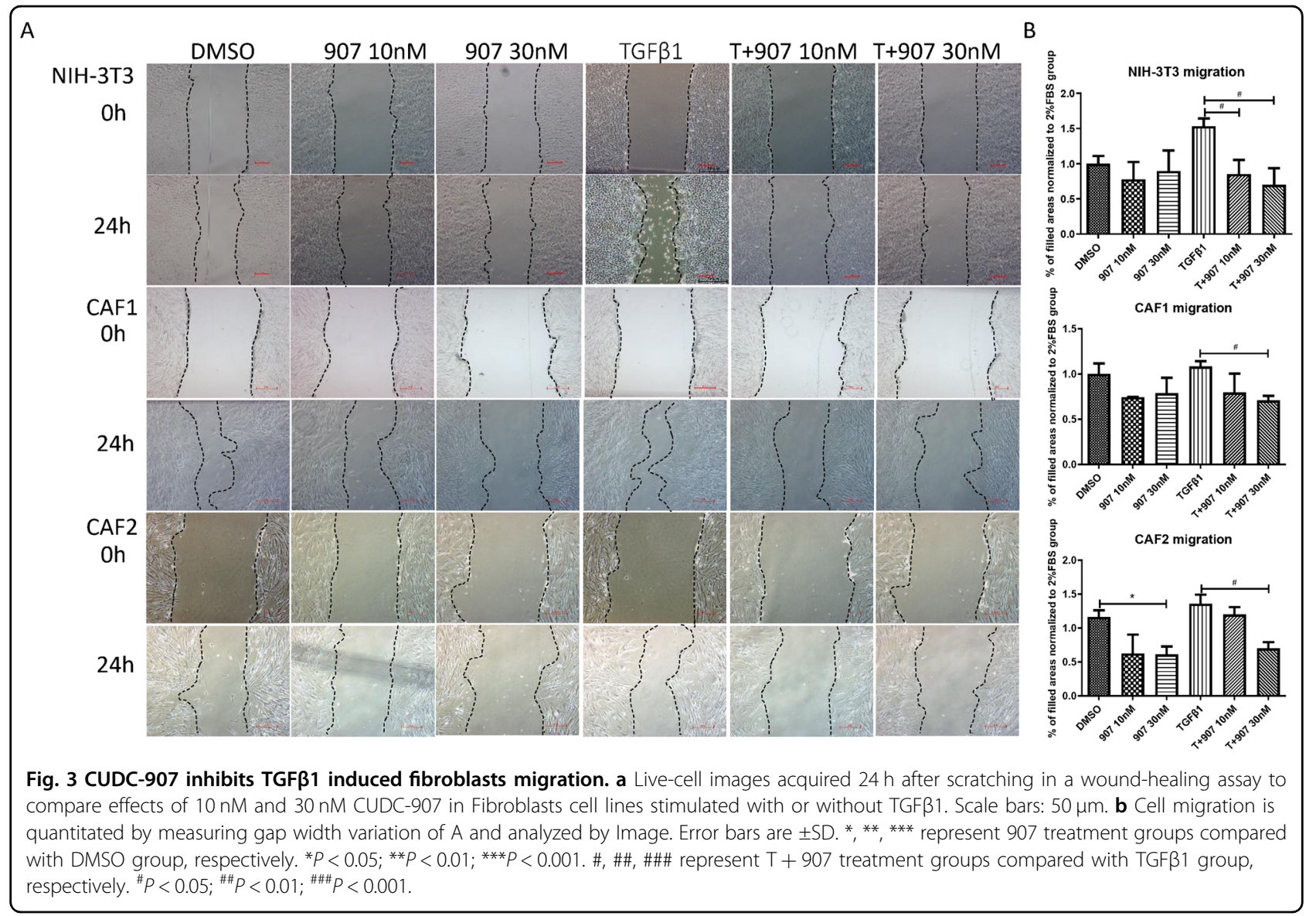

suppressing inflammation and epithelial proliferation made it not a good idea of global inhibition of TGF $\beta 1$ signaling ${ }^{27}$. It has been reported that PI3K-AKT signaling pathways are commonly induced in myofibroblasts by TGF $\beta 1$ and targeting mTOR mediates many physiological functions such as metabolism, cell cycle progression, proliferation, migration, and also contributes to collagen accumulation ${ }^{28}$. PI3K activation results in production of membrane-localized phosphatidylinositol3,4,5-trisphosphate $\left(\mathrm{PIP}_{3}\right)$ and lead mTOR functions at two distinct nodes in this axis. mTOR complex1 (mTORC1) signaling induces canonical Smad activation via $\mathrm{p} 70 \mathrm{~S} 6 \mathrm{~K}$ and $4 \mathrm{E}-\mathrm{BP} 1$ phosphorylation which strongly mediates the fibro genic effects of TGF $\beta 1$. mTOR complex 2 (mTORC2) and 3-phosphoinositide-dependent protein kinase-1(PDK1) phosphorylate Akt (Ser473) and SGK1 that leads to human lung fibroblasts proliferation and differentiation ${ }^{29,30}$. The reduction of gene expression of TGF- $\beta 1$ and abrogation of its downstream cascade may, thus, elucidate why CUDC-907 showed such potent antifibrotic capacity ${ }^{31}$.

HDACs maintain a dynamic equilibrium in the cell in conjunction with histone acetyl transferases, which deacetylate lysine residues on histones to bring about transcriptional expression. TGF $\beta 1$-induced myofibroblasts activation was accompanied by a general reduction in histone acetyltransferases (HAT), and divergent changes in histone deacetylase (HDAC) enzymes at both transcript and protein levels ${ }^{32}$. The activities of all Class I and II HDACs were reported to be significantly upregulated in IPF lung tissues ${ }^{33}$. Many studies have used HDAC inhibitors to block lung fibrotic process and tumor growth, and SAHA, a nonselective HDAC Class I and II inhibitor, has been reported to have the ability to inhibit the differentiation of TGF $\beta 1$-induced myofibroblasts ${ }^{34-36}$. Similarly, Trichostatin A, a recently proved HDAC inhibitor, works at a micromolar level and sufficiently inhibits fibroblasts in vitro ${ }^{17}$. However, increased apoptosis resistance of myofibroblasts results in cellular resistance to certain HDACIs ${ }^{33}$. For example, HDAC2 is increased in the middle and late stages of bleomycin-induced lung fibrosis in mice ${ }^{37}$ and is associated with resistance to Fasmediated apoptosis ${ }^{38}$. Thus, monotherapy with agents that target a driver event is not likely to yield durable responses. Thus, the effective inhibition of multiple pathways active in myofibroblasts with CUDC-907 treatment, i.e. its effect as an HDAC and PI3K inhibitor, suggest that it may be more effective than monotherapy. 


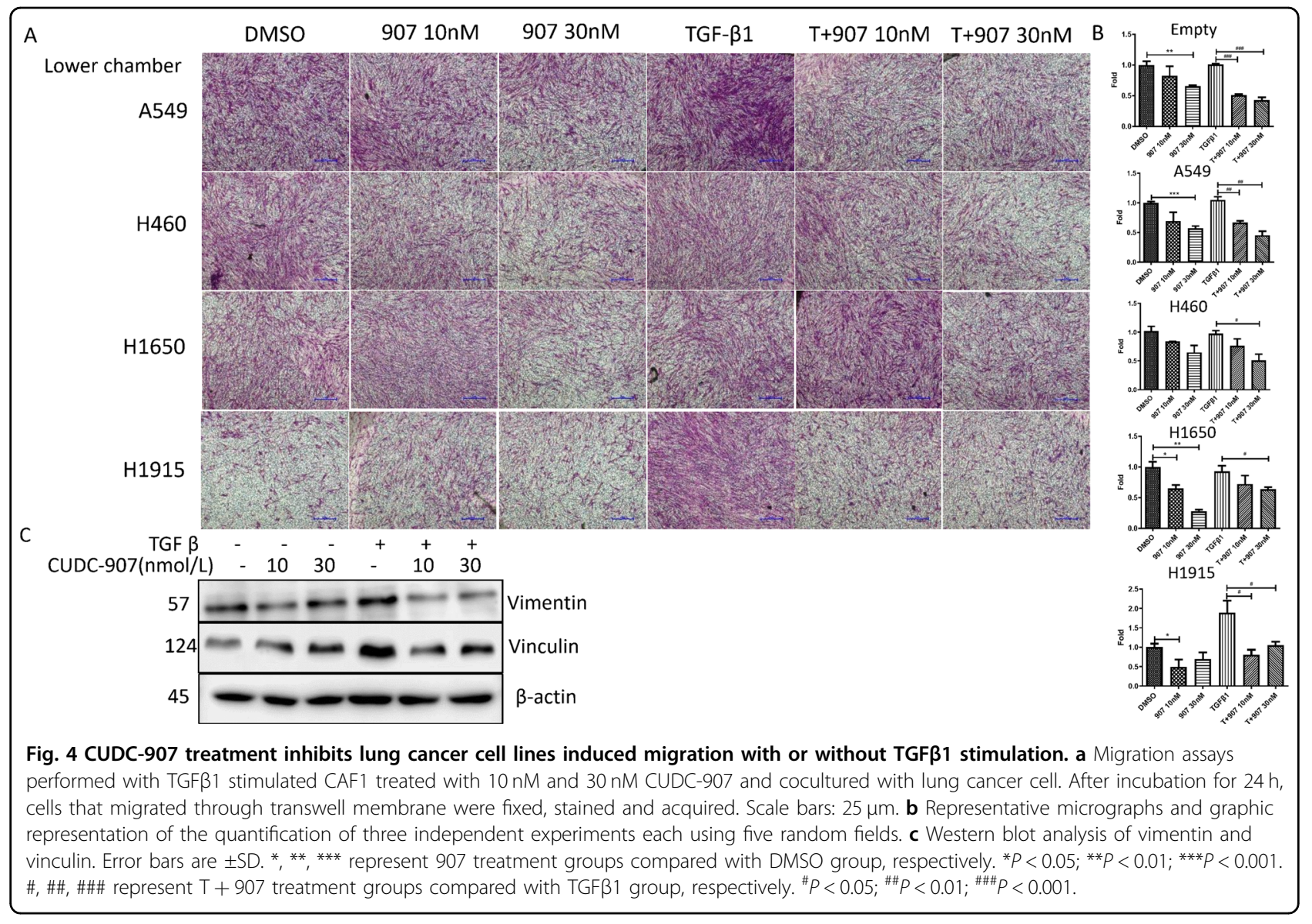

So far it has been shown that suppression of histone deacetylase and phosphoinositide 3-kinase simultaneously worked better than single HDAC inhibitor for the treatment of cutaneous $\mathrm{T}$-cell lymphoma or other cancers ${ }^{39,40}$. Besides, a hybrid molecule like CUDC-907 becomes difficult in escaping from cells after being converted into its pharmacologically-active acid form so that its cell concentration easily build-up ${ }^{41}$. Therefore, this newlysynthesized small molecular compound designed as a dual inhibitor of PI3K/Akt/mTOR and HDAC, came into our sight and was tested in this study. In our studies to understand the mechanism of action of CUDC-907 on cellular proliferation, migration, and invasion, and target HDACs, we have identified that $30 \mathrm{nM}$ of CUDC-907 could potently suppress migration and invasion ability of TGF $\beta 1$-induced fibroblasts and CAF as well as their ability of collagen production. Moreover, we compared the inhibitory effect of CUDC-907, GDC-0941, and Trichostatin $\mathrm{A}$ at $30 \mathrm{nM}$ in this research and confirmed that CUDC-907 inhibited myofibroblasts better in cell proliferation, indicating that CUDC-907 may be a better option for lung fibrotic disease therapy.

Such similar phenomena were also reported in previous published investigations. Different anti-proliferative mechanisms of mTOR or HDAC inhibitor have been reported, such as G0-G1 and G2-M cell cycle arrest, or induced apoptosis ${ }^{11,42-45}$. Previous study revealed that CUDC-907 induced a G2/GM cell cycle arrest of H460, a human tumor cell line ${ }^{19}$, indicating that CUDC-907 may interfere in cell mitosis. We found that CUDC-907 causes cell-cycle arrest at G0/G1, followed by activation of apoptosis post treatment. This effect was also reported in human bone marrow stromal cells when exposed to CUDC- $907^{46}$. Kotian et al. ${ }^{23}$ discovered that CUDC-907 induced G2-M arrest and apoptosis by downregulating cyclin B1, AURKA, AURKB, and PLK1 while upregulating p21 and p27 levels. However, the mechanism of CUDC907 in regulating G0-G1 arrest is still unknown. Simultaneously, the attenuated ability of cell migration and invasion in myofibroblasts was relevant to the inhibition of PI3K pathway as well as the function of HDAC ${ }^{47,48}$. The anticancer effects of CUDC-907, such as attenuation of migration and invasion were realized partially by induction of TWIST1 and E-cadherin expression. However, the main mechanism remains to be explored.

As an oral, first-in-class and rationally-designed, compound, CUDC-907 was recently applied in a phase-I clinical trial of relapsed or refractory lymphoma or 

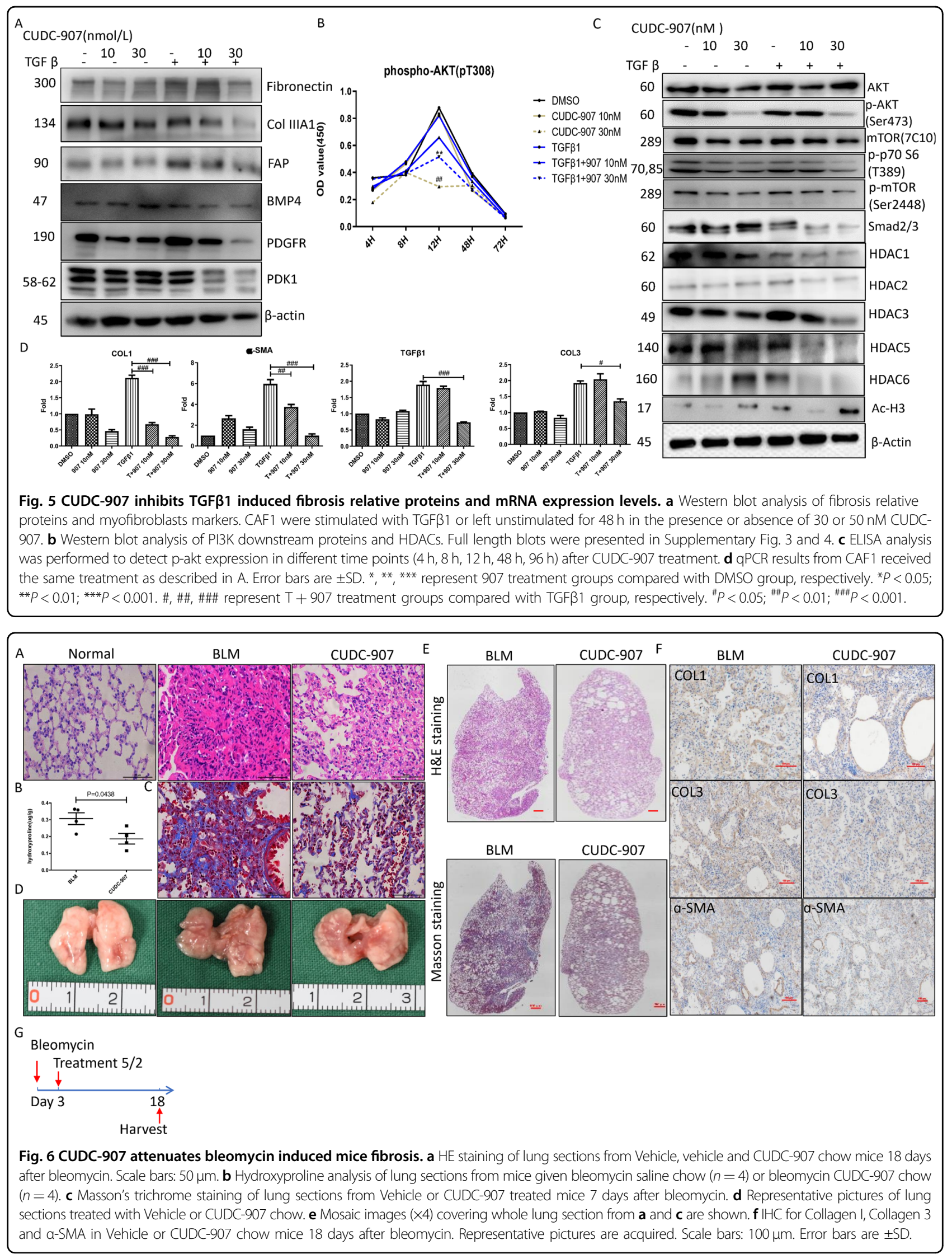


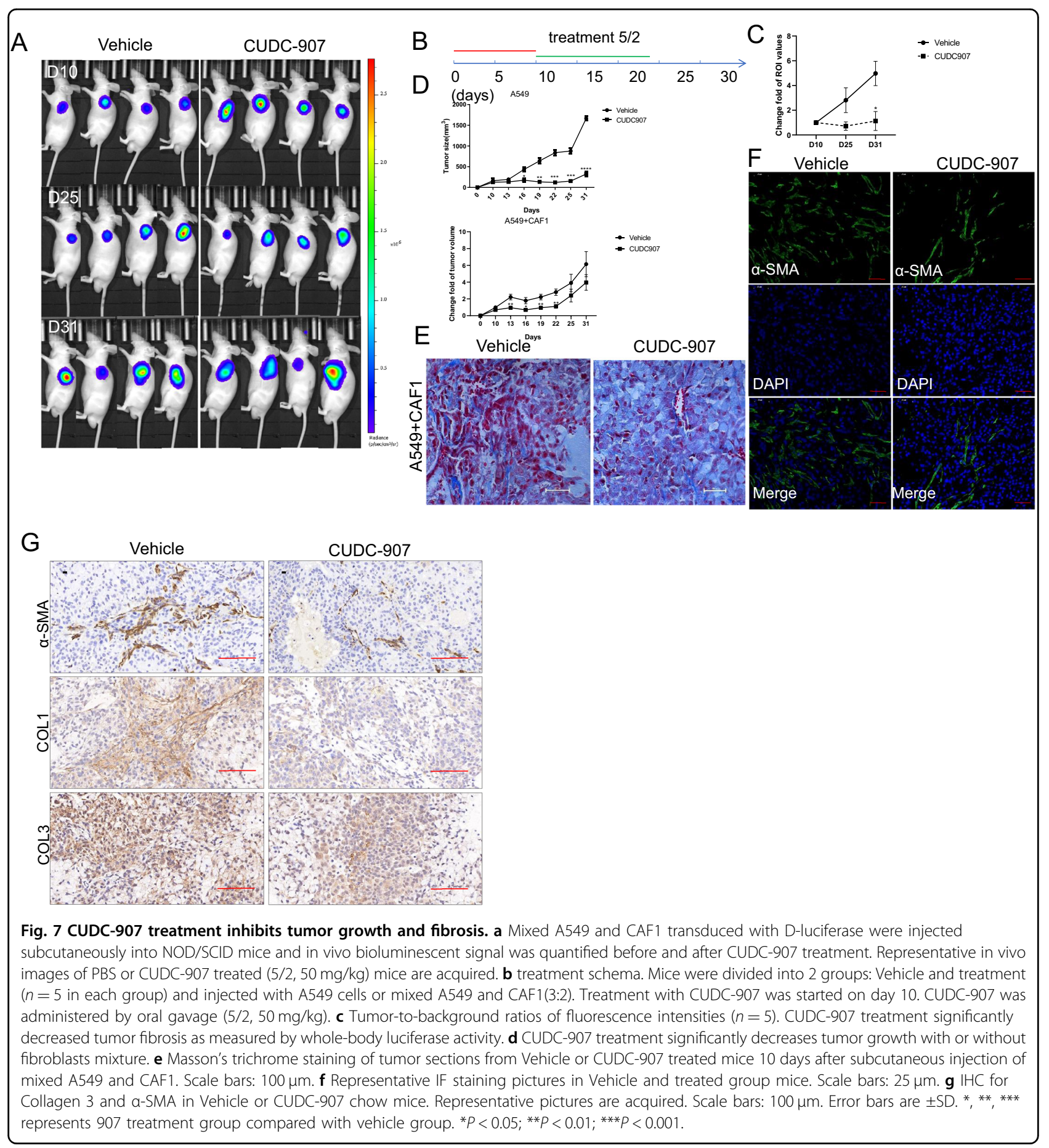

multiple myeloma, that revealed its potential value in future clinical application for cancer therapy ${ }^{20}$. Furthermore, the US FDA has granted its orphan drug designation for the treatment of patients with Diffuse Large B-cell Lymphoma. The role of CUDC-907 in targeting myofibroblasts revealed in the current study may suggest that CUDC-907 could potentially be used as a drug for fibrosis and cancer therapy with further confirming studies on clinical efficacy and biosafety.

In summary, we confirmed the efficient inhibition of both PI3K/Akt/mTOR and HDAC by CUDC-907, and therefore demonstrated its potent counteractive role in suppressing proliferation, collagen production and ECM deposition, and inhibiting the properties of migration and 
invasion of fibroblasts and CAF. All of these represent a therapeutic alternative strategy for lung fibrosis and cancer that should be investigated in a clinical future.

\section{Acknowledgements}

This study was financially supported by China National Nature Science Foundation (81871882), Science and Technology Commission of Shanghai Municipality Medical Guidance Science \& Technology Support Project (16411966100), Shanghai Municipal Education Commission-Gaofeng Clinical Medicine Grant Support (20172005) and Shanghai Municipal Commission of Health and Family Planning Outstanding Academic Leaders Training Program (2017BR055)

\section{Author details \\ 'Department of Thoracic Surgery, Ruijin Hospital, Shanghai Jiao Tong University School of Medicine, 197 Ruijin 2nd Rd, Shanghai 200025, China. Department of Plastic and Reconstructive Surgery, Shanghai Ninth People's Hospital, Shanghai Jiao Tong University School of Medicine, Shanghai Key Laboratory of Tissue Engineering, 639 ZhiZaoJu Road, Shanghai 200011, China. ${ }^{3}$ Renji-Med X Clinical Stem Cell Research Center, Renji Hospital, School of Medicine, Shanghai Jiao Tong University, No. 160 Pu-Jian Road, Shanghai 200127, China}

\section{Conflict of interest}

The authors declare that they have no conflict of interest.

\section{Publisher's note}

Springer Nature remains neutral with regard to jurisdictional claims in published maps and institutional affiliations.

Supplementary Information accompanies this paper at (https://doi.org/ 10.1038/s41419-020-02916-w).

Received: 8 January 2020 Revised: 5 May 2020 Accepted: 6 May 2020 Published online: 17 September 2020

\section{References}

1. Thannickal, V. J. et al. Fibrosis: ultimate and proximate causes. J. Clin. Invest 124, 4673-4677 (2014).

2. Kaminski, N. et al. Global analysis of gene expression in pulmonary fibrosis reveals distinct programs regulating lung inflammation and fibrosis. Proc. Natl Acad. Sci. USA 97, 1778-1783 (2000).

3. Saito, A., et al., The role of TGF-beta signaling in lung cancer associated with idiopathic pulmonary fibrosis. Int. J. Mol. Sci. 19, 3611-3625 (2018).

4. Cruz-Bermudez, A. et al. Cancer-associated fibroblasts modify lung cancer metabolism involving ROS and TGF-beta signaling. Free Radic. Biol. Med. 130, 163-173 (2019).

5. Gallego-Munoz, P. et al. Effects of TGFbeta1, PDGF-BB, and bFGF, on human corneal fibroblasts proliferation and differentiation during stromal repair. Cytokine 96, 94-101 (2017).

6. Baarsma, H. A. et al. Glycogen synthase kinase-3 (GSK-3) regulates TGF-beta(1)induced differentiation of pulmonary fibroblasts. Br. J. Pharm. 169, 590-603 (2013).

7. Li, S. et al. The fibroblast TIAM2 promotes lung cancer cell invasion and metastasis. J. Cancer 10, 1879-1889 (2019).

8. Alguacil-Nunez, C. et al. Current perspectives on the crosstalk between lung cancer stem cells and cancer-associated fibroblasts. Crit. Rev. Oncol. Hematol. 125, 102-110 (2018)

9. Watanabe, R., Wei, L. \& Huang, J. mTOR signaling, function, novel inhibitors, and therapeutic targets. J. Nucl. Med. 52, 497-500 (2011).

10. Li, J. et al. Rictor/mTORC2 signaling mediates TGFbeta1-induced fibroblast activation and kidney fibrosis. Kidney Int 88, 515-527 (2015).

11. Syed, F. et al. Potent dual inhibitors of TORC1 and TORC2 complexes (KU0063794 and KU-0068650) demonstrate in vitro and ex vivo anti-keloid scar activity. J. Invest Dermatol 133, 1340-1350 (2013).

12. Fitzgerald O'Connor, E. J. et al. Histone deacetylase 2 is upregulated in normal and keloid scars. J. Invest. Dermatol. 132, 1293-1296 (2012).
13. Mutze, $\mathrm{K}$. et al. Histone deacetylase (HDAC) 1 and 2 expression and chemotherapy in gastric cancer. Ann. Surg. Oncol. 17, 3336-3343 (2010).

14. Ouaissi, M. et al. Further characterization of HDAC and SIRT gene expression patterns in pancreatic cancer and their relation to disease outcome. PLoS One 9, e108520 (2014)

15. Marks, P. A. et al. Histone deacetylase inhibitors. Adv. Cancer Res. 91, 137-168 (2004).

16. Van Beneden, $\mathrm{K}$. et al. HDAC inhibitors in experimental liver and kidney fibrosis. Fibrogenes. Tissue Repair 6, 1 (2013).

17. Diao, J. S. et al. Trichostatin A inhibits collagen synthesis and induces apoptosis in keloid fibroblasts. Arch. Dermatol. Res. 303, 573-580 (2011).

18. Mondello, P. et al. Dual inhibition of histone deacetylases and phosphoinositide 3-kinase enhances therapeutic activity against B cell lymphoma. Oncotarget 8, 14017-14028 (2017).

19. Qian, C. et al. Cancer network disruption by a single molecule inhibitor targeting both histone deacetylase activity and phosphatidylinositol 3-kinase signaling. Clin. Cancer Res. 18, 4104-4113 (2012).

20. Younes, A. et al. Safety, tolerability, and preliminary activity of CUDC-907, a firstin-class, oral, dual inhibitor of HDAC and PI3K, in patients with relapsed or refractory lymphoma or multiple myeloma: an open-label, dose-escalation, phase 1 trial. Lancet Oncol. 17, 622-631 (2016).

21. Yi, Y. et al. Cancer-associated fibroblasts promote epithelial-mesenchymal transition and EGFR-TKI resistance of non-small cell lung cancers via HGF/IGF1/ANXA2 signaling. Biochim Biophys. Acta Mol. Basis Dis. 1864, 793-803 (2018).

22. Wang, W. et al. Induction of predominant tenogenic phenotype in human dermal fibroblasts via synergistic effect of TGF-beta and elongated cell shape. Am. J. Physiol. Cell Physiol. 310, C357-C372 (2016).

23. Kotian, S. et al. Dual inhibition of HDAC and tyrosine kinase signaling pathways with CUDC-907 inhibits thyroid cancer growth and metastases. Clin. Cancer Res. 23, 5044-5054 (2017)

24. Ivaska, J. et al. Novel functions of vimentin in cell adhesion, migration, and signaling. Exp. Cell Res. 313, 2050-2062 (2007)

25. Humphries, J. D. et al. Vinculin controls focal adhesion formation by direct interactions with talin and actin. J. Cell Biol. 179, 1043-1057 (2007).

26. Rahman, A., et al., Vinculin regulates directionality and cell polarity in 2D, 3D matrix and 3D microtrack migration. Mol. Biol. Cell 27, 1431-1441 (2016).

27. Akhurst, R. J. \& Hata, A. Targeting the TGFbeta signalling pathway in disease. Nat. Rev. Drug Discov. 11, 790-811 (2012).

28. Orani, A. et al. [Multidisciplinary study of 2 cases of herpetic encephalitis]. Boll. Ist. Sieroter. Milan. 62, 62-71 (1983).

29. Laplante, M. \& Sabatini, D. M. mTOR signaling in growth control and disease. Cell 149, 274-293 (2012).

30. Garcia-Martinez, J. M. \& Alessi, D. R. mTOR complex 2 (mTORC2) controls hydrophobic motif phosphorylation and activation of serum- and glucocorticoid-induced protein kinase 1 (SGK1). Biochem. J. 416, 375-385 (2008).

31. Bettinger, D. A. et al. The effect of TGF-beta on keloid fibroblast proliferation and collagen synthesis. Plast. Reconstr. Surg. 98, 827-833 (1996).

32. Smith, E. R. et al. TGF-beta1 modifies histone acetylation and acetyl-coenzyme A metabolism in renal myofibroblasts. Am. J. Physiol. Renal. Physiol. 316 F517-F529 (2019)

33. Korfei, M. et al. Aberrant expression and activity of histone deacetylases in sporadic idiopathic pulmonary fibrosis. Thorax 70, 1022-1032 (2015).

34. Wang, Z. et al. Suberoylanilide hydroxamic acid: a potential epigenetic therapeutic agent for lung fibrosis?. Eur. Respir. J. 34, 145-155 (2009).

35. Lyu, X. et al. HDAC inhibitors as antifibrotic drugs in cardiac and pulmonary fibrosis. Ther. Adv. Chronic Dis. 10, 2040622319862697 (2019).

36. Korfei, M. et al. Comparison of the antifibrotic effects of the pan-histone deacetylase-inhibitor panobinostat versus the IPF-drug pirfenidone in fibroblasts from patients with idiopathic pulmonary fibrosis. PLoS One 13, e0207915 (2018).

37. Li, M. et al. Effects of dynamic changes in histone acetylation and deacetylase activity on pulmonary fibrosis. Int Immunopharmacol. 52, 272-280 (2017).

38. Huang, S. K. et al. Histone modifications are responsible for decreased Fas expression and apoptosis resistance in fibrotic lung fibroblasts. Cell Death Dis. 4, e621 (2013).

39. Pei, Y. et al. HDAC and PI3K antagonists cooperate to inhibit growth of MYCdriven medulloblastoma. Cancer Cell 29, 311-323 (2016).

40. Wozniak, M. B. et al. Vorinostat interferes with the signaling transduction pathway of T-cell receptor and synergizes with phosphoinositide-3 kinase inhibitors in cutaneous T-cell lymphoma. Haematologica 95, 613-621 (2010). 
41. Delcuve, G. P., Khan, D. H. \& Davie, J. R. Targeting class I histone deacetylases in cancer therapy. Expert Opin. Ther. Targets 17, 29-41 (2013).

42. Kawada, J. et al. mTOR inhibitors induce cell-cycle arrest and inhibit tumor growth in Epstein-Barr virus-associated T and natural killer cell lymphoma cells. Clin. Cancer Res. 20, 5412-5422 (2014).

43. Cuyas, E. et al. Cell cycle regulation by the nutrient-sensing mammalian target of rapamycin (mTOR) pathway. Methods Mol. Biol. 1170, 113-144 (2014).

44. Loos, C. et al. Amino-functionalized nanoparticles as inhibitors of mTOR and inducers of cell cycle arrest in leukemia cells. Biomaterials 35, 1944-1953 (2014).
45. Du, L. et al. A potent HDAC inhibitor, 1-alaninechlamydocin, from a Tolypocladium sp. induces G2/M cell cycle arrest and apoptosis in MIA PaCa-2 cells. J. Nat. Prod. 77, 1753-1757 (2014).

46. Ali, D. et al. CUDC-907 promotes bone marrow adipocytic differentiation through inhibition of histone deacetylase and regulation of cell cycle. Stem Cells Dev. 26, 353-362 (2017).

47. Ramakrishnan, S. et al. HDAC 1 and 6 modulate cell invasion and migration in clear cell renal cell carcinoma. BMC Cancer 16, 617 (2016).

48. Sun, Z., Cao, B. \& Wu, J. Protease-activated receptor 2 enhances renal cell carcinoma cell invasion and migration via PI3K/AKT signaling pathway. Exp. Mol. Pathol. 98, 382-389 (2015). 